\title{
Ma chi sono questi norreni?
}

\author{
Enzo Caffarelli
}

PUBBLICATO: 30 APRILE 2019

\section{Quesito:}

Alcuni lettori ci chiedono se il termine norreno sia utilizzabile solo in riferimento alla lingua e alla letteratura o possa estendersi anche ad altri aspetti culturali di quella popolazione (artigianato norreno, invasioni norrene, tradizioni norrene ecc.).

\section{Ma chi sono questi norreni?}

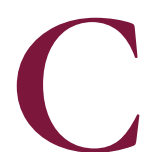

on norreno, dal nordico norron 'settentrionale', che il GRADIT data al I954 con la marca TS indicante voce "tecnico-specialistica", s'intende per il GDLI tutto ciò "che si riferisce alla lingua e alla letteratura norvegese e islandese al IX al XIV sec., cioè nell'età vichinga”, con la seguente specificazione: "e il termine è impiegato per designare globalmente le culture sviluppatesi in Norvegia, in Islanda e nelle colonie minori dell'Atlantico settentrionale e, in partic., le forme e i generi letterari comuni a tale area geografica, come il carme eddico, il carme scaldico e la saga; si può ricordare in proposito che la saga dei Nibelunghi [...]".

Il Vocabolario Treccani online è più dettagliato e aggiunge che tali opere ci permettono "di conoscere la mitologia, la religione, la vita dell'antico mondo germanico al culmine del suo sviluppo storico e alla vigilia del passaggio al cristianesimo. In partic. sono noti come saghe $n$. anonimi racconti in prosa fissati per iscritto, soprattutto in Islanda, a partire dal sec. $13^{\circ}$, dopo una lunga tradizione orale, e aventi per tema i fatti salienti della colonizzazione dell'isola e della storia norvegese che a essi è collegata".

Quanto alla prima presunta attestazione in italiano, una ricerca su Google Libri consente di retrodatare norreno almeno al 1894 , anno di pubblicazione del saggio Letteratura norvegiana del catanese Santi Consoli (Milano, Ulrico Hoepli). Tra le occorrenze nel testo si legge norreno/-a/-e/-i associato a poesia II volte (più una a poeti), a lingua Io, poema/-i 5, tradizioni 5, prosa/-e 3, letteratura 3 , miti/mitologia 3, versificazione/verso 3, voce/-i 2, eroi I, skaldi I (intesi come poeti itineranti di corte in corte in era vichinga); 8 volte norreno è glottonimo, una volta infine vale come etnico. Da segnalare che i norreni sono indicati come "gli odierni norvegiani".

È pertanto evidente e del tutto naturale che l'aggettivo norreno riguarda tutto quanto sappiamo del mondo norreno e che alcune popolazioni del Nord Europa hanno ereditato, coltivano e studiano: la lingua, certo, che alcuni considerano collocarsi rispetto alle parlate scandinave moderne come il latino alle lingue romanze; ma anche la cultura in generale, la storia, la religione, usi e costumi.

E di là da fatti linguistici, culturali in senso lato (si parla spesso di "eredità norrena") e storici, troviamo in rete rare attestazioni anche di "mare norreno", "fiordi norreni", "pianure norrene", "terra, landa, costa norrena", dunque voci d'àmbito geografico, e inoltre derivati occasionali come norrenocentrico (in un blog dove il termine norreni viene usato come sinonimo di 'polentoni, milanesi, italiani del nord' e altro ancora, denotando una notevole confusione e pur tuttavia testimoniando una certa familiarità con la voce...). 
Infine norreno può essere utilizzato in funzione suppletiva anche in tempi moderni, come sinonimo in particolare di norvegese e di islandese. Lo incontriamo in rete, sia pure in casi rarissimi, in ambito sportivo. Nessuna meraviglia, se si considera quanti suppletivi da etnici o toponimi/oronimi/idronimi antichi popolano, e ancor più popolavano nel XX secolo, le cronache soprattutto calcistiche per amor divariatio. Tuttora non è inconsueto leggere o ascoltare lusitani per 'portoghesi', magiari per 'ungheresi', ellenici per 'greci', elvetici per 'svizzeri'.

\section{Cita come:}

Enzo Caffarelli, Ma chi sono questinorreni?, "Italiano digitale", 2019, IX, 2019/2 (aprile-giugno)

DOI: $10.35948 / 2532-9006 / 2020.3110$

Copyright 2019 Accademia della Crusca

Pubblicato con licenza creative commons CC BY-NC-ND 